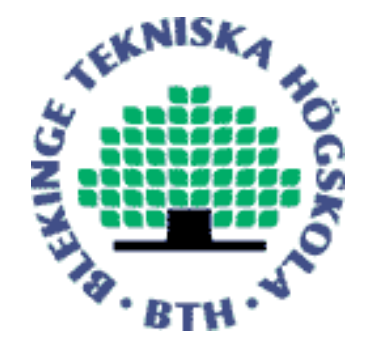

Copyright (C) 2011 IEEE.

Citation for the published paper:

Toward Cost-Efficient Integration of Telematic Systems Using K-Spanning Tree and Clustering Algorithms

Gideon Mbiydzenyuy, Jan Persson, Paul Davidsson

14th International IEEE Annual Conference on Intelligent Transportation Systems Washington DC, USA, 2011

2011 Washington DC

This material is posted here with permission of the IEEE. Such permission of the IEEE does not in any way imply IEEE endorsement of any of BTH's products or services Internal or personal use of this material is permitted. However, permission to reprint/republish this material for advertising or promotional purposes or for creating new collective works for resale or redistribution must be obtained from the IEEE by sending a blank email message to pubs-permissions@iee.org.

By choosing to view this document, you agree to all provisions of the copyright laws protecting it. 


\title{
Toward Cost-Efficient Integration of Telematic Systems Using K-Spanning Tree and Clustering Algorithms
}

\author{
Gideon Mbiydzenyuy ${ }^{1}$, Jan A. Persson ${ }^{2}$, and Paul Davidsson ${ }^{3}$ \\ ${ }^{1}$ Email: gideon.mbiydzenyuy@bth.se \\ ${ }^{2}$ Email: jan.persson@bth.se \\ ${ }^{3}$ Email: paul.davidsson@bth.se \\ ${ }^{123}$ Blekinge Institute of Technology/Computing, Biblioteksgatan 4, 37424 Karlshamn, Sweden
}

\begin{abstract}
This article uses analytical methods to assess reductions in total costs of telematic systems that can result from common infrastructure utilization. Analytical methods based on clustering and K-minimum spanning tree can be adopted for finding clusters or sets which maximize reductions in total system costs due to infrastructure sharing between telematic systems. Efficient integration of telematic systems through infrastructure sharing can positively influence telematic service interoperability while reducing costs. Results show the measure of synergy for each K-value, as well as total cost savings of up to $2 \%$.
\end{abstract}

\section{INTRODUCTION}

Several years of experimentation and research in Information and Communication Technology (ICT) has led to systems (telematic systems e.g. Navigation) offering services with the potential to improve transport system performance on safety and the environment [1]-[3]. High costs (among other reasons) make it difficult to realize the full potential of telematic systems and services that will improve transport systems [4]. This article uses analytical methods to assess reductions in total cost of telematic systems that can result from common infrastructure utilization. The common use of functionality by telematic systems can be regarded as sharing the underlying infrastructure providing such functionality. Multiple telematic systems can share infrastructure, leading to cost synergies, and ultimately a decrease of total system costs [5]. Analytical methods based on clustering and $\mathrm{K}$-minimum spanning tree (K-MST) can be adopted for finding clusters or sets which maximize reductions in total system costs due to infrastructure sharing between telematic systems. The results can lead to cost-efficient integration of telematic systems in platforms that promote the sharing of system infrastructure.

Transport Telematic Services (TTSs) result from deployment of expensive infrastructure e.g. wireless GSM communication and digital map positioning infrastructure [6]. Such TTSs are considered as an added value by consumers, who perceive the costs of such TTSs as additional costs to transport services and hence expensive [7]. Subsidies, developments of low- cost technology e.g. for communication, and efficient integrated use of telematic system infrastructure are all mea- sures that can contribute to low-cost, affordable TTSs. Efficient integration of telematic systems through infrastructure sharing can further have a positive influence on telematic service interoperability while reducing costs. The need for efficient sharing of infrastructure resources is addressed by several research works such as Intelligent Road Infrastructure [8], assessment of multi-service architectures [9], service bundling analysis [1], electric vehicle service packages [2] etc. Some of these approaches are inspired by existing information system platforms like the Internet and use of APIs (Application Programming Interfaces). Hence, several attempts have aimed to benefit from potential synergies as a result of sharing infrastructure functionalities such as GSM communication and digital map positioning.

To efficiently integrate TTSs through shared infrastructure requires that, for each TTS, we assess its functionalities and possible reduction in total cost as a result of infrastructure sharing. Such assessments should point to variation of synergy for sets (or clusters) of TTSs that share infrastructure. One major advantage of such sets of TTSs is the ability to achieve reduction in total cost, leading to improved cost efficiency regarding system integration. In addition to information on reductions in total cost, there are other aspects that need to be taken into consideration in order to achieve efficient integration of telematic systems e.g. societal benefits. We chose to focus on the cost reduction when TTSs share infrastructure as opposed to individual implementation. We further ignore the value (benefits to society) of different TTSs so as to concentrate on the costs. In this article, we extend a previously proposed method of synergy analysis [9] by carrying out experiments of how to maximize reductions in total system cost of multiple TTSs that can lead to cost- efficient integration of such TTSs. Given a set of TTSs, we apply an analytical method to determine the similarity between all possible pairs of TTSs. The reduction in total cost between pairs of TTSs is used as a measure of synergy between such TTSs. The result is a symmetric matrix of pair-wise similarities between TTSs. Hierarchical clustering is used for finding sets of TTSs with high synergies. A minimum spanning tree problem (MSTP) 
involves finding a spanning tree $\left(D^{*} \subseteq S\right)$ of an undirected, connected graph $(\mathrm{G})$ such that the sum of the weights of the selected edges is minimum [10], [11] . This problem has been widely studied and several variations researched, such as Kleast weight spanning tree, K-best possible spanning tree [7] etc. TTSs can be considered as the vertices of a tree with reduction in costs between a pair of TTSs (when both are included in the set) taken as the edges. Thus, we use a Kminimum spanning tree algorithm for finding the sets of TTSs which (approximately) maximize reductions in total system costs or synergy value. $\mathrm{K}$ represents the maximum number of TTSs allowed in a set.

The data used includes a specification of the costs for each functionality used by each TTS and an assessment of the percentage of cost reduction factor $\left(\beta_{j}\right)$ of the functionality $(\mathrm{j})$ when shared by at least two TTSs (see Appendix). The results of applying K-MST algorithm (K-MSTA) show the measure of synergy for each K-value. The results indicate a total cost savings of up to $2 \%$. The rest of this paper consists of the following sections (section numbers in brackets): Methodology of synergy analysis for cost-efficient integration of telematic systems (II), Experiment set-up (III), Results and analysis (IV), Conclusion and future work (V). These are followed by an acknowledgment, references and an appendix.

\section{METHODOLOGY: SYNERGY ANALYSIS FOR COST-EFFICIENT INTEGRATION OF TELEMATIC SYSTEMS}

\section{A. Conceptual description}

One way to specify TTSs so as to analyze the cost-efficiency of shared infrastructure utilization is by considering their functionality specification. When combined together and with appropriate input, system functionalities will result in a given system behavior. Examples of such functionalities may range from digital map positioning and GSM communication to various data collection sensors. Primary system requirements are met with a specific implementation of system functionalities. In addition to total costs of functionalities for each TTS, there are overhead costs such as labor, administration and in some cases regulation of standards may also add costs. Conceptually,

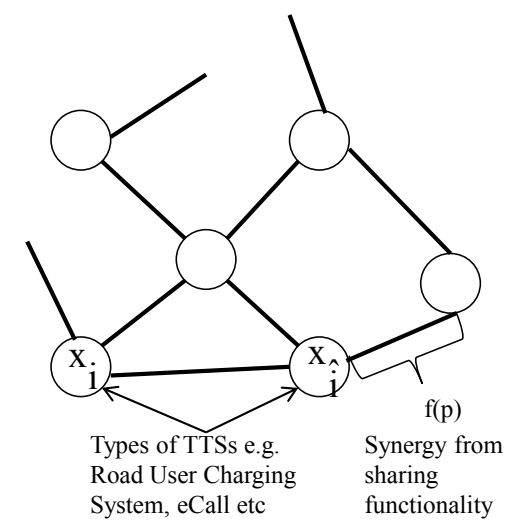

Fig. 1: Concept of shared infrastructure between TTSs the relationship between TTSs (e.g. $x_{i}$ and $x_{\hat{i}}$ ) can be simplified in pair-wise relations as illustrated by Figure 1. One basic way to relate these pairs is to estimate the percentage reduction in costs if the two TTSs share infrastructure. We refer to such a measure as synergy, following previous similar analysis [9]. By using such a synergy assessment, potential TTSs can be identified for cost-efficient integration. Sets of TTSs with high cost reduction potential can be integrated together through shared system infrastructure, hence minimizing total system costs.

In a previous study an approach is suggested for analysis of synergy between sets of TTSs (denoted by $\mathrm{S}$ ) based on an anticipated cost reduction factor (denoted by $\beta_{j}$ ) for each functionality $j[9]$. In the present study, we apply a synergy measure concerning the ratio of the (net) reduction in total costs due to synergy, to the total costs without synergy, as shown by Equation 1 (i.e. percentage reduction).

$$
f(p)=\frac{U_{p}-V_{p}}{U_{p}}
$$

where $\mathrm{p}$ is the pair-wise elements of TTSs in set S. $U_{p}$ and $V_{p}$ are based on the fixed costs of the functionalities as follows: $U_{p}$ is total system cost (assuming independent implementation, i.e. no infrastructure sharing) and can never be zero and $V_{p}$ is the reduced costs with infrastructure sharing. $V_{p}$ is calculated from the assessment of reduction between TTSs $\left(\beta_{j}\right)$ for each functionality $\mathrm{j}$. Equation 1 can be seen as a synergy measure for all functionalities of a pair of TTSs. To calculate the synergy for the complete set of TTSs, Hierarchical Clustering Algorithm (HCA) and K-MST are employed. The input to the following two algorithms is the pair-wise measurements between TTSs based on Equation 1. The outputs of the algorithms are synergy measurements for sets of TTSs with varying cardinality (see result and analysis section below).

\section{B. Nearest neighbor hierarchical agglomeration (HCA)}

Agglomerative algorithms begin by placing distinct data points in a cluster (i.e. $D_{i}=x_{i}: i=1 \cdots n$ ) and successively merging the different clusters to form a bigger cluster based on some similarity measure until all elements are contained in a single cluster $\left(D=\cup D_{i}: i=1 \cdots n\right)$. The similarity measure is the ratio of the (net) reduced costs due to synergy, to the total costs without synergy (Equation 1), and defines the distance between two clusters. The pseudo code for the algorithm is shown below $(n=|S|)$ :

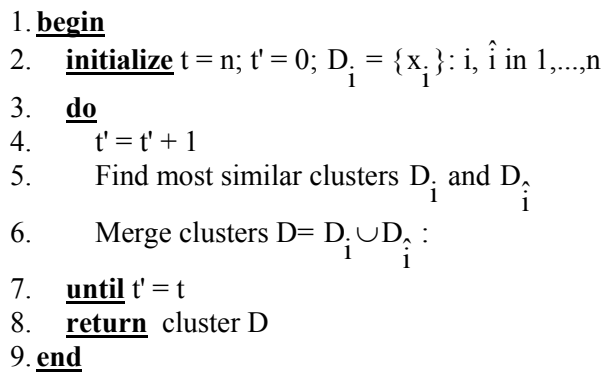


In this study, we use complete linkage for the similarity measure. In complete linkage, the maximum distance between any two elements in each of the clusters defines the distance between the clusters i.e. the most similar cluster is obtained by $d_{\max }\left(D_{i}, D_{\hat{i}}\right)=\max \left\|x_{i}-x_{\hat{i}}\right\|$, where $x_{i} \in D_{i}$ and $x_{\hat{i}} \in$ $D_{\hat{i}} . x_{i}$ and $x_{\hat{i}}$ represent different TTSs, $d_{\max }$ is calculated by Equation 1 (above).

\section{K-Minimum Spanning Tree Algorithm (K-MSTA)}

Let $\mathrm{G}=(\mathrm{S}, \mathrm{E}, \mathrm{w})$ be an undirected, edge-weighted graph with $|S|=n$ and $|E|=m$ denoting the number of nodes and edges of $\mathrm{G}$. The function

$$
w: E \rightarrow \mathbb{R}^{+}, w\left(E^{\prime}\right)=\sum_{e \in E^{\prime}} w(e)
$$

denotes the edge-weight function for any $E^{\prime} \subset E$. Consider the following definition: a cardinality sub graph is a connected sub graph D of G: $|E(D)|=K$. The K-cardinality sub graph problem is to find a K-cardinality sub graph of minimal weight i.e. $\min \{w(E(D))$ :D is a K-cardinality sub graph $\}$. For a MSTP, $D=G$ and $K=n-1$ and for a K-MSTP $D \subset G$ and $2 \leq K \leq n-1$. For a given value of $\mathrm{K}$ (as input), the K-MSTP can be solved in polynomial time [12].

In order to study the potential total savings for a given set of TTSs (denoted by S) with cardinality n, we propose and use a variant of the K-minimum spanning tree algorithm formulated as an Integer Linear program [12] . For any number of TTSs K, the aim is to determine the total savings potential (by solving the MSTP for $\mathrm{K}$ ) due to synergies. K-value is the maximum allowed number of TTSs in the set currently considered. The result is a tree (set of TTSs) that has the maximum total synergy. The proposed pseudo code for the algorithm is as follows:

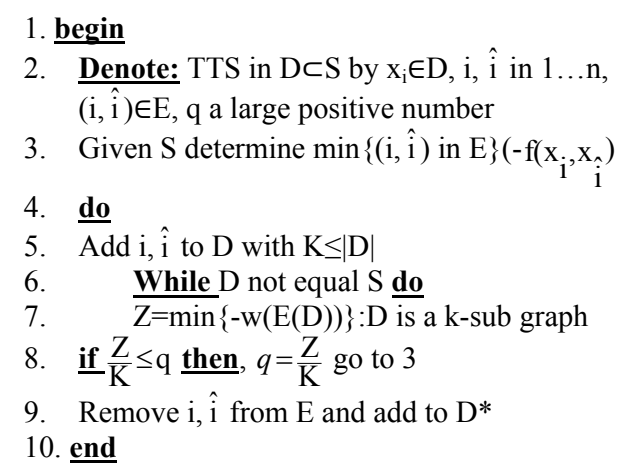

There are no cycles in the final graph and all the weights are positive. The function $\mathrm{f}(\mathrm{p})$ measures the synergy (weight between a pair of TTSs) in the interval $0 \leq f(p)<1$ and is equivalent to the percentage gain. Observe that we only consider the cases where $V_{p}$ is non-zero $\left(V_{p}=0\right.$ means $\mathrm{f}(\mathrm{p})$ is 1) because in practical situations, shared infrastructure utilization can lead to a reduction in costs $\left(V_{p}<U_{p}\right)$ or no reduction at all $\left(U_{p}=V_{p}\right)$, but not to zero costs, hence the interval $0 \leq f(p)<1$. The solution consists of edges in the improving direction and hence the proposed algorithm can be seen to be greedy.

\section{EXPERIMENT SET-UP}

Input data to the experiment is obtained from different sources as shown in Table 1.

\begin{tabular}{|l|l|l|c|}
\hline Transport Telematic Services & $\begin{array}{l}\text { Related } \\
\text { Project }\end{array}$ & Year & Source \\
\hline $\begin{array}{l}\text { Automated Driver Logs } \\
\text { (ADL) }\end{array}$ & ABECQ & $\begin{array}{l}(01- \\
08) 2008\end{array}$ & {$[13]$} \\
\hline $\begin{array}{l}\text { Accident Warning Informa- } \\
\text { tion (AWI) }\end{array}$ & CODIA & $\begin{array}{l}(01- \\
08) 2008\end{array}$ & {$[4]$} \\
\hline E-Call (EC) & e-IMPACT & $2006-2007$ & $\begin{array}{c}{[5],} \\
{[14]}\end{array}$ \\
\hline $\begin{array}{l}\text { Intelligent Speed Adaptation } \\
\text { (ISA) }\end{array}$ & $\begin{array}{l}\text { UK-ISA, } \\
\text { e-IMPACT }\end{array}$ & $2006-2007$ & $\begin{array}{c}{[5],} \\
{[15]}\end{array}$ \\
\hline $\begin{array}{l}\text { Navigation Through a Route } \\
\text { Network (NAV) }\end{array}$ & $\begin{array}{l}\text { Tom-Tom } \\
\text { website }\end{array}$ & 2011 & {$[16]$} \\
\hline $\begin{array}{l}\text { On-Board Safety and Secu- } \\
\text { rity Monitoring (OSM) }\end{array}$ & ECORYS & $2005-2006$ & {$[17]$} \\
\hline $\begin{array}{l}\text { Real Time Track and Trace } \\
\text { of Goods (RTT) }\end{array}$ & AVL-Transit & $1998-1999$ & {$[18]$} \\
\hline $\begin{array}{l}\text { Road Hindrance Warning } \\
\text { (RHW) }\end{array}$ & CODIA & $\begin{array}{l}(01- \\
08) 2008\end{array}$ & {$[4]$} \\
\hline Road User Charging (RUC) & ARENA & $2007-$ \\
\hline present & {$[19]$} \\
\hline Route Guidance (RG) & $\begin{array}{l}\text { Tom-Tom } \\
\text { website }\end{array}$ & 2011 & {$[16]$} \\
\hline $\begin{array}{l}\text { Sensitive Goods Monitoring } \\
\text { (SGM) }\end{array}$ & $\begin{array}{l}\text { GOOD } \\
\text { ROUTE }\end{array}$ & $2006-2009$ & {$[20]$} \\
\hline $\begin{array}{l}\text { Theft Alarm and Recovery } \\
\text { (TAR) }\end{array}$ & $\begin{array}{l}\text { TM Fleet } \\
\text { website }\end{array}$ & 2011 & {$[21]$} \\
\hline Weight Indication (WI) & Thesis Project & 2007 & {$[22]$} \\
\hline
\end{tabular}

TABLE 1: Input data obtained from different project sources.

In total, 13 TTSs (13 vertices, 12 edges, i.e. no cycles) are studied (Table 1). Each TTS is specified by a set of functionalities (26 in total). Common to all TTSs are the following functionalities: digital map (exception: ADL), wireless communication device with antenna and SIM card, graphical interface (exception: ISA, RUC), docking station and labor or installation cost. All data are converted to the cost equivalent for the year 2011 with discount rate $=5 \%$ and distributed over a time period of 10 years (life span of functionality). Using the data, we construct pair-wise synergies of TTSs. In addition to the functionalities specified for each TTS from different sources, anticipated functionalities have been included where necessary, e.g. we consider the possibility to communicate information between vehicle and roadside equipment for a Weigh-in-motion service, which is not often the case for existing systems reported in the literature.

In some situations we use different decomposition techniques in order to estimate the fixed costs of a functionality. For instance, by studying the price variation between different versions of a telematic system (each with different functionalities), additional costs between versions can be approximated to be the cost of such new functionalities. As an example consider a basic car navigation system (NAV1) with total cost $100 €$, consisting of a digital map, route suggestion estimates based on daily traffic (IQ route), a functionality for reporting 


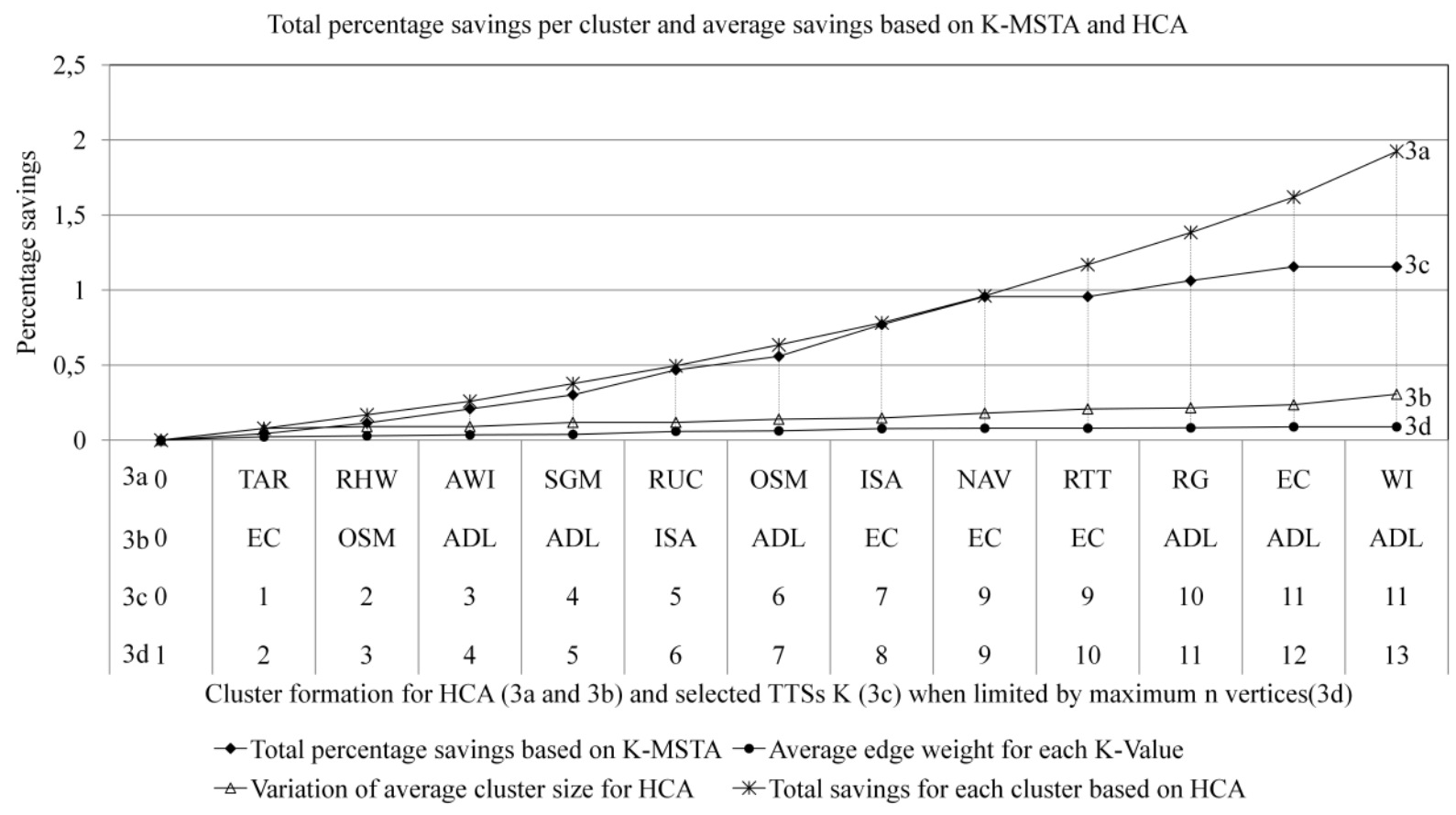

Fig. 2: Results of complete linkage HCA ( $3 a$ and $3 b$ ) and K-MST algorithms (3c and 3d) both showing total cost savings for each set of TTS (3a and 3c) and average cost savings with each additional TTS link (3b and 3d).

map changes, SIM for communication, LCD interface and a docking station. Assume that a second version called NAV2 is then released with a total cost of $120 €$ with the additional functionality of advanced lane guidance and text-to-speech. From this it can be deduced within a certain degree of accuracy that the advanced lane guidance and text-to-speech functionalities will share the total additional cost of about $20 €$. How such costs are distributed between the functionalities is not trivial. In the absence of any source of information, we followed an average distribution of costs between different functionalities resulting from a given component as illustrated below:

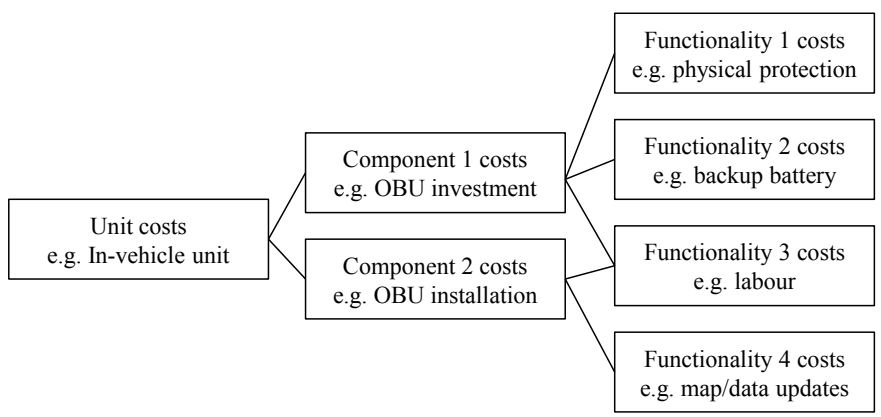

Fig. 3: Illustration of cost distribution between functionalities.

HCA is implemented using XLMiner an Excel add-in and K-MSTA is implemented in a mathematical programming environment called AMPL.

\section{RESULTS AND ANALYSIS}

Results of the HCA indicate the order of final cluster formation where the TTSs TAR and EC are most likely to have the highest possible savings, and WI and ADL show the least savings. Cumulative addition of savings per link shows a steady growth as in Figure 2. Such cumulative additions do not show much information about the total savings associated with a cluster of a given size. While the results are true for the final cluster, the curve showing total savings maybe different if the algorithm is applied to different subsets of TTSs. For studying such variations we consider the K-MSTA.

A general tendency for HCA is the increase in total synergy with additional TTSs (3a). Since HCA is based on pairwise synergies, there is an accuracy problem (due to double counting) estimating the synergy for a set of TTSs. This is the reason why HCA is showing larger savings compared to the K-MSTA. The results of the K-MSTA show the estimated total synergy for each K-value (3c) to be generally less when compared to HCA. The addition of each TTS in a set leads to increased synergy both in average savings (3b) per TTS and in total savings (3c) up to about 9 TTSs. This is partly due to the choices of TTS used in this experiment which turn to have similarities with each other and hence synergies.

Both approaches show a fairly similar trend, where the cumulative total savings of the final cluster formed by HCA reaches $1.9 \%$ and the final cluster for K-MSTA reaches $1.1 \%$. The two approaches are based on different criteria for evaluating similarity where higher savings generally means higher similarity. The validity of the results above (Figure 2) depends on a number of factors, e.g. the total number 
of TTSs and functionalities considered, the total number of users etc. As we studied only 13 TTSs with 26 functionalities and an individual user situation, the results of the percentage savings are low (less than $2 \%$ ). The savings are likely to be higher with a large number of users, TTSs and functionalities taken into consideration. Because K-MSTA is heuristic, the question on the optimal K-Value remains open. Our focus was estimating the cost reduction rather than seeking to achieve an optimal implementation of the K-MSTA. Even though the KMSTA may provide more accurate cost reduction estimates, the challenge is in dealing with a large set of TTSs as the power set will result in a huge search space.

\section{CONCLUSION AND FUTURE WORK}

This paper has focused on assessment of cost reduction when TTSs share infrastructure as opposed to individual implementation. The results of applying K-MSTA show the measure of synergy for each K-value that can lead to total cost savings up to $2 \%$. Hierarchical clustering is used to determine variation in synergy in a given set of TTSs. To determine the synergy measure for a given number of TTSs (K), we apply a variant of K-minimum spanning tree algorithm. Based on data from different sources (Table 1), the synergy variation for different collections of TTSs is a step toward cost-efficient integration of telematic systems. Such results can be useful for the analysis of system designs by influencing the choices of systems to consider. The main challenge remains information regarding absolute system cost, which is seldom available and where available is old and outdated. This information is important for research related to cost efficiency (in integration of TTSs) and even cost benefit assessments.

Key elements of this study that we consider for further improvements are: refinements of cost reduction potential $\left(\beta_{j}\right)$, refinement of cost per functionality, consideration of the positive benefits of the TTSs, further refinement of the KMSTA and additional data concerning TTSs. The value of the cost reduction potential $\left(\beta_{j}\right)$ used in this study is currently based on the views of the authors. The value for some functionalities is a non-linear variation and depends on the number of applications. By monitoring results of new experiments related to implementation of TTSs and discussions with other experts more accurate estimates can be obtained.

\section{ACKNOWLEDGMENT}

This research work is funded by the Swedish Government Agency for Innovation (http:/www.vinnova.se) and the National Swedish Transport Administration (Trafikverket: http://www.trafikverket.se/).

\section{APPENDIX}

See Table 2

\section{REFERENCES}

[1] B. Grush, "Make boring on-board units for road pricing interesting." Busan, South Korea: Proceedings of the 17th World Congress on Intelligent Transport Systems, 2010.
[2] C. Hays and V. Kaul, "Strategic market and technology assessment of telematic applications for electric vehicles." MI, USA: Proceedings of the 10th annual telematics conference, exhibition, award, TELEMATICS DETROIT, 2010.

[3] E. SAMPSON, "Wireless technologies and the transformation of transport." LEIPZIG, Germany: International Transport Forum: TRANSPORT AND INNOVATION, 2010.

[4] Risto Kulmala, Pekka Leviäkangas, Niina Sihvola, Pirkko Rämä, Jonathan Francsics, Ewan Hardman, Simon Ball, Bob Smith, Ian McCrae, Tim Barlow and Alan Stevens, "Co-operative systems deployment impact assessment (codia), final study report, deliverable 5," VTT Technical Research Centre of Finland, Under EU, DG-INFSO, Tech. Rep., 2008.

[5] Kerry Malone, Isabel Wilmink, Gerhard Noecker, Konrad Rorucker, Roland Galbas, Tom Alkim, "eIMPACT: Socio-economic impact assessment of stand-alone and co-operative intelligent vehicle safety systems (iVSS) in europe, final report of iVSS, v2 deliverable d10," eIMPACT consortium, EU Sixth Framework Programme ., Tech. Rep., 2008.

[6] Eurobarometer, "Use of intelligent systems in vehicles," European Commission, Field Work, Special Eurobarometer 267, Tech. Rep., 2006.

[7] David Eppstein, "Finding the k smallest spanning trees," In 2nd Scandanavian Workshop on Algorithm Theory, 2011.

[8] F. op de Beek, P. van der Kroon and R.Kulmala, "Final report and recommendations of the intelligent infrastructure working group v1.0," eSafety Forum Directorate-General Information Society of the European Commission, Tech. Rep., 2010.

[9] G. Mbiydzenyuy, "Assessment of telematic systems for road freight transport (licentiate thesis)," Blekinge Institute of technology, Sweden, Jan. 2011.

[10] C. F. Bazlamacca and K. S. Hindi, "Minimum-weight spanning tree algorithms a survey and empirical study," Computers and Operations Research, vol. 28, pp. 767-785, 2001

[11] H. J. Greenberg, "Greedy algorithms for minimum spanning tree," University of Colorado at Denver, Tech. Rep., 1998.

[12] H. H. Ehrgott M, Freitag J and M. F., "Heuristics for the k-cardinality tree and subgraph problem." Asia-Pacifc Journal of Operational Research, vol. 14, pp. 87-114, 1997

[13] ABECQ, "Alcolock version 3, webpage," http://www.abecq.com/ AlcolockV3 EN.html, last accessed 2011-04-09, 2011.

[14] D McClure and A Graham, "ecall the case for deployment in the uk," SBD under a contract with the Transport Technology and Standards Division of the DfT., Tech. Rep., 2006.

[15] Oliver Carsten, Frank Lai, Kathryn Chorlton, Paul Goodman, David Carslaw and Stephane Hess, "Speed limit adherence and its effects on road safety and climate change, final report, ISA," UK Commission for Integrated Transport, Tech. Rep., 2008

[16] Tom-Tom, "Navigation systems, webpage," http://www.tomtom.com/en gb/products/car-navigation/, last accessed 2011-04-09, 2011.

[17] ECORYS, "Cost-benefit assessment and prioritisation of vehicle safety technologies framework contract REN/A1/56-2004 lot 2: Economic assistance activities final report ref: TREN-ECON2-002," European Commission - DG TREN, Tech. Rep., 2006.

[18] Zhong-Ren Peng, Edward A. Beimborn, Simi Octania and Richard J. Zygowicz, "Evaluation of the benefits of automated vehicle location systems in small and medium sized transit agencies," Wisconsin Department of Transportation, Tech. Rep., 1999.

[19] Jonas Sundberg, "Kilometerskatt för tunga lastfordon i sverige. reviderad kostnadsbedömning," ARENA Project Internal document, Tech. Rep. 2007.

[20] Michael Landwehr and Florian Krietsch, "GOOD ROUTE, CBA and CEA on developed applications, dangerous goods transportation routing, monitoring and enforcement, deliverable 9.3, IST-4-027873-STREP," INFORMATION SOCIETY TECHNOLOGIES (IST) PROGRAMME, Tech. Rep., 2007.

[21] TM-Fleet, "Heavy equipment tracking and theft recovary system, webpage," http://tmfleet.com/onlinestore/index.php?main_page=product info\&cPath=1\&products_id=2\&zenid=42ie235sop5m6a4bhjr0u2b034, last accessed 2011-04-09, 2011.

[22] L. Zhang, "An evaluation of the technical and economic performance of weigh-in-motion sensing technology," Master's thesis, Waterloo University, Ontario, Canada, 2007. 


\begin{tabular}{|c|c|c|c|c|c|c|c|c|c|c|c|c|c|c|}
\hline $\begin{array}{l}\text { Functionalities, j (Rows) } \\
\text { and TTSs, i (Columns) }\end{array}$ & $\beta_{j}$ & ADL & AWI & EC & ISA & NAV & OSM & RTT & RHW & RUC & RG & SGM & TAR & WI \\
\hline $\begin{array}{l}\text { In-band modern license } \\
\text { (TBC) }\end{array}$ & 0.40 & & & 18.23 & & & & & & & & & & \\
\hline Back-up GSM antenna & 0.50 & & & 18.23 & & & & & & & & & & \\
\hline Backup battery & 0.10 & 30.00 & & 18.23 & & 25.00 & & & & & 30.00 & & 60.00 & 0.11 \\
\hline Hardware and software & 0.70 & 666.50 & & 18.23 & & 30.00 & & & & 18.23 & 30.00 & & 25.00 & 0.20 \\
\hline $\begin{array}{l}\text { Interface and cover pro- } \\
\text { tection }\end{array}$ & 0.50 & 10.00 & 687.58 & 18.23 & & 35.00 & 906.16 & & 161.15 & 233.06 & 35.00 & 176.40 & 68.00 & \\
\hline Manual eCall button & 0.20 & & & 12.76 & 112.35 & & & & & & & & & \\
\hline GSM antenna & 0.40 & & 2630.00 & 25.53 & 112.35 & 50.00 & & & 893.86 & & 50.00 & 176.40 & 100.00 & \\
\hline Microphone & 0.30 & & & 12.76 & & 20.00 & & & & & 20.00 & & & \\
\hline $\begin{array}{l}\text { Wiring harness and con- } \\
\text { nectors }\end{array}$ & 0.10 & & & 18.23 & 112.35 & & & & & & & & & \\
\hline $\begin{array}{l}\text { Embedded SIM card for } \\
\text { communication }\end{array}$ & 0.50 & 27.00 & 739.15 & 38.29 & 193.41 & 27.00 & 193.41 & 193.41 & 292.00 & 193.41 & 27.00 & 176.40 & 27.00 & 193.41 \\
\hline $\begin{array}{l}\text { Vehicle manufacturers } \\
\text { development costs }\end{array}$ & 0.20 & & 957.21 & 229.73 & 189.00 & & 315.00 & & & & & & & \\
\hline Processor (RAM) & 0.30 & & & & 112.35 & & & & 955.95 & 18.23 & & 176.40 & & 0.07 \\
\hline map updates & 0.40 & & 72.60 & 72.60 & 12.60 & 90.00 & 72.60 & & 72.60 & 72.60 & 50.00 & 137.81 & & 72.60 \\
\hline $\begin{array}{l}\text { Speed limit and exter- } \\
\text { nal roadside control e.g. } \\
\text { cameras }\end{array}$ & 0.20 & & & & 50.40 & & & & 1020.63 & 19.45 & & 275.63 & & \\
\hline Labor/installation costs & 0.20 & 88.13 & 88.13 & 88.13 & 94.50 & 88.13 & 88.13 & 0.40 & 88.13 & 194.48 & 88.13 & 137.81 & & 13.46 \\
\hline $\begin{array}{l}\text { Compiling digital map } \\
\text { of } 1 \mathrm{KM} \text { road length }\end{array}$ & 0.30 & & & & 23.09 & & & & & & & & & \\
\hline Back office & 0.40 & & & & & & & 0.60 & & 19.45 & & 551.25 & & \\
\hline System control point & 0.08 & & & & & & & & & 27.96 & & 8.48 & & \\
\hline DSRC & 0.33 & & 687.58 & & & & & & 161.15 & & & 176.40 & & \\
\hline In-vehicle OBU & 0.20 & & & & & 20.00 & & 0.08 & & 223.65 & 60.00 & 330.75 & & \\
\hline Sensors & 0.10 & 666.50 & 608.48 & & & & 315.00 & & 96.47 & & & & & 0.47 \\
\hline Data logger & 0.20 & & & & & & 127.63 & & & & & & & 0.11 \\
\hline In-vehicle camera & 0.30 & & & & & 10.00 & & & & & & & & \\
\hline Auto SMS capability & 0.10 & & & & & & & & & & & & 68.00 & \\
\hline Alarm system & 0.30 & & & & & & & & & & & & 68.00 & \\
\hline $\begin{array}{l}\text { Docking station (con- } \\
\text { nectors) Tom Tom }\end{array}$ & 0.50 & 50.00 & 50.00 & & 50.00 & 50.00 & 50.00 & 50.00 & 50.00 & 50.00 & 50.00 & 50.00 & 50.00 & 50.00 \\
\hline Cost per TTS in $€$ & & 1538.13 & 6520.74 & 589.20 & 1062.40 & 445.13 & 2067.93 & 244.48 & 3791.94 & 1070.52 & 440.13 & 2373.73 & 466.00 & 330.44 \\
\hline Data Reference & & {$[13]$} & \begin{tabular}{l|l}
$4]$ & \\
\end{tabular} & {$[14]$} & \begin{tabular}{l|l}
{$[15]$} & \\
\end{tabular} & {$[16]$} & [17] & {$[18]$} & \begin{tabular}{l|l}
{$[4]$} & \\
\end{tabular} & [19] & {$[16]$} & {$[20]$} & {$[21]$} & {$[22]$} \\
\hline
\end{tabular}

TABLE 2: Input data employed in the experiment (from Table 1). All cost values are in $€$. The rows represent functionalities (with the exception of the last two rows that indicates the total costs per TTS and data references respectively) and columns represent the TTSs (with the exception of columns one and two that represent functionality name and assessments of infrastructure sharing potential for at least two TTSs $\left(\beta_{j}\right)$ respectively). An empty cell implies the TTS does not require functionality. 